\title{
Découverte fortuite d'un cas de COVID-19 par TEP-TDM
}

\author{
Reshma Amin MD, Leonard Grinblat MD, Mansoor Husain MD
}

- Citation : CMAJ 2020 June 8;192:E631. doi : 10.1503/cmaj.200831-f; diffusion hâtive le 22 mai 2020

Voir la version anglaise de l'article ici : www.cmaj.ca/lookup/doi/10.1503/cmaj.200831

$\mathbf{U}$ ne femme diabétique et obèse morbide (indice de masse corporelle : $44,3 \mathrm{~kg} / \mathrm{m}^{2}$ ) de 58 ans se présente en consultation externe pour la stadification habituelle de son lymphome hodgkinien. L'intervention se fait par tomographie par émission de positrons combinée à la tomodensitométrie (TEP-TDM). Évaluée avant d'entrer dans la clinique, la patiente dit ne pas avoir de symptômes de la maladie à coronavirus 2019 (COVID-19), ne fait pas de fièvre, n'a pas voyagé récemment et n'a pas été en contact avec une personne atteinte. On lui injecte du fluorodésoxyglucose, un analogue du glucose marqué par un isotope radioactif du fluor, le fluor-18 (18F-FDG), puis elle reste en décubitus dorsal dans la salle d'injection pour faciliter la distribution physiologique du produit. Pendant ce temps, le personnel remarque qu'elle a des quintes de toux occasionnelles.

La TEP-TDM confirme que la patiente présente une adénopathie pelvienne de stade 2 du côté droit (indiquée par la flèche blanche à la figure $1 \mathrm{~A}$ ), comme l'avait fait la biopsie, mais l'activité du ${ }^{18}$ F-FDG n'y est que faible (valeur de fixation normalisée maximale [SUVmax] : 2,9). L'examen révèle également des opacités multifocales périphériques aux 2 poumons (figures $1 C$ et $1 D$ ), et une activité modérée du ${ }^{18} \mathrm{~F}$-FDG (SUVmax : 4,5) dans le lobe inférieur gauche (indiquée par la flèche blanche à la figure 1C). Nous n'avons relevé aucun épanchement pleural ni adénopathie médiastinale avide de ${ }^{18 F-F D G . ~ L a ~ t o m o d e n s i t o m e ́ t r i e ~ d i a g n o s t i q u e ~ d e ~ s t a d i f i c a t i o n ~ e f f e c-~}$ tuée une semaine plus tôt au niveau du thorax ne montrait aucune opacité (figure 1B).

Nous avertissons immédiatement le médecin traitant. La patiente est dirigée vers la clinique désignée afin de subir un test de dépistage du coronavirus du syndrome respiratoire aigu sévère 2 (SRAS-CoV-2) le jour même, et reçoit la consigne de s'isoler chez elle. Le test, qui se fait par amplification en chaîne par polymérase couplée à une transcription inverse (RT-PCR), est déclaré positif 2 jours plus tard. La patiente a maintenant le nez qui coule, tousse plus souvent et fait de la fièvre $\left(38^{\circ} \mathrm{C}\right)$.

Beaucoup de personnes qui se sont présentées en consultation externe pour des examens d'imagerie diagnostique après un
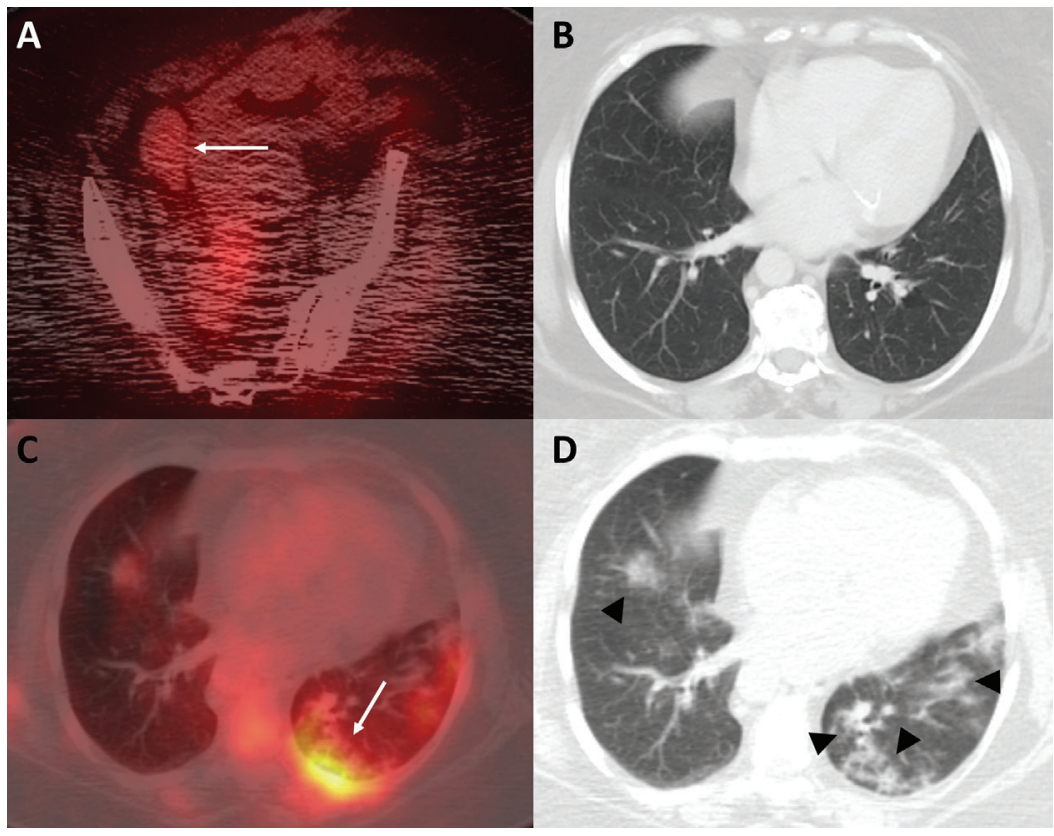

Figure 1. (A) Image fusionnée en coupe axiale obtenue par tomographie par émission de positrons combinée à la tomodensitométrie (TEP-TDM), qui montre la fixation du fluorodésoxyglucose marqué au fluor 18 ( $\left.{ }^{18} \mathrm{~F}-\mathrm{FDG}\right)$ sur un lymphome situé du côté droit du pelvis (flèche blanche) d'une femme de 58 ans. (B) Image obtenue par tomodensitométrie thoracique une semaine avant la visite en clinique, sur laquelle on voit bien la base des poumons. (C) Image née en coupe axiale obtenue par TEP-TDM, qui montre des infiltrats multifocaux bilaté列 mon gauche (flèche blanche). (D) Image correspondante non fusionnée, obtenue seulement par tomodensitométrie, qui montre des infiltrats multifocaux bilatéraux (triangles noirs).

court dépistage de la COVID-19 ont par la suite montré des anomalies inattendues à la radiographie et à la tomodensitométrie thoraciques ${ }^{1}$, typiquement des opacités en verre dépoli multifocales ou des infiltrats plus denses². À la TEP-TDM, la pneumonie associée à la COVID-19 démontre une avidité pour le ${ }^{18} \mathrm{~F}_{-\mathrm{FDG}^{3-5}}$. Certains chercheurs croient que l'activité du ${ }^{18} \mathrm{~F}-\mathrm{FDG}$ pourrait avoir une valeur pronostique, mais pour l'instant la littérature scientifique se limite à des études de $\mathrm{cas}^{3}$.

La patiente est finalement hospitalisée et gardée en observation. Ses symptômes s'estompent dans la semaine qui suit, après quoi elle reçoit son congé. Ses prochaines radiographies pelviennes sont reportées en raison des restrictions dues à la pandémie de COVID-19. 


\section{Références}

1. Pozzessere C, Rotzinger DC, Ghaye B, et al. Incidentally discovered COVID-19 pneumonia: the role of diagnostic imaging [lettre]. Eur Radiol 4 mai 2020 [Cyberpublication avant impression]. doi: 10.1007/s00330-020-06914-6.

2. Shi H, Han X, Jiang N, et al. Radiological findings from 81 patients with COVID-19 pneumonia in Wuhan, China: a descriptive study. Lancet Infect Dis 2020; 20:425-434.

3. Deng Y, Lei L, Chen Y, et al. The potential added value of FDG PET/CT for COVID-19 pneumonia. Eur J Nucl Med Mol Imaging le 21 mars 2020 [Cyberpublication avant impression]. doi: 10.1007/s00259-020-04767-1.

4. Albano D, Bertagna F, Bertolia M, et al. Incidental findings suggestive of COVID19 in asymptomatic patients undergoing nuclear medicine procedures in a high prevalence region. J Nucl Med 2020;61:632-6.

5. Lütje S, Marinova M, Kütting D, et al. Nuclear medicine in SARS-CoV-2 pandemia: 18F FDG-PET/CT to visualize COVID-19. Nuklearmedizi le 7 avril 2020 [Cyberpublication avant impression]. doi: 10.1055/a-1152-2341.
Intérêts concurrents : Leonard Grinblat et Mansoor Husain sont actionnaires minoritaires de l'établissement privé (le Centre MyHealth de Toronto) où ce cas a été vu. Aucun autre intérêt concurrent n'a été déclaré.

Cet article a été révisé par des pairs.

Les auteurs ont obtenu le consentement de la patiente.

Affiliations : Hôpital SickKids (Amin); Hôpital général de North York (Grinblat); Centre MyHealth (Grinblat); Réseau universitaire de santé (Husain), Toronto, Ont.

Remerciements : Les auteurs remercient le Dr Marc Freeman (Centre MyHealth, Toronto) pour son aide dans l'interprétation des images obtenues par tomographie par émission de positrons combinée à la tomodensitométrie.

Correspondance : Leonard Grinblat, I.grinblat@myhealthcentre.ca 